\title{
Invasive ductolobular carcinoma of the breast: spectrum of mammographic, ultrasound and magnetic resonance imaging findings correlated with proportion of the lobular component
}

\author{
Gisela LG Menezes ${ }^{1 *}$, Maurice AAJ van den Bosch', Emily L Postma², Mary-Ann El Sharouni², \\ Helena M Verkooijen ${ }^{2}$, Paul J van Diest $^{3}$ and Ruud M Pijnappel ${ }^{1}$
}

\begin{abstract}
Purpose: The aim of this study was to describe the imaging features of patients with invasive ductolobular carcinoma of the breast in comparison with the proportion of the lobular component.

Materials and methods: We retrospectively reviewed mammographic, sonographic and MRI records of 113 patients with proven ductolobular carcinoma diagnosed between January 2008 and October 2012 according to the BI-RADS ${ }^{\otimes}$ lexicon, and correlated these to the proportion of the lobular component.

Results: At mammography the most common finding (62.9\%) for invasive ductolobular carcinoma was an irregular, spiculated and isodense mass. On ultrasound an irregular and hypoechoic mass, with spiculated margins and posterior acoustic shadowing was observed in $46.8 \%$ of cases. Isolated mass and mass associated with non-mass like enhancement (NMLE) were the most common findings by MRI (89.4\%). Washout pattern in delayed phase was seen in $61.2 \%$ and plateau curve was more frequently observed in patients with larger lobular component. Additional malignant findings (multifocality, multicentricity and contralateral disease) did not correlate significantly with the proportion of the lobular component.
\end{abstract}

Conclusion: Invasive ductolobular carcinoma mainly presents as an irregular, spiculated mass, isodense on mammography and hypoechoic with posterior acoustic shadowing. On MRI it is usually seen as an isolated mass or as a dominant mass surrounded by smaller masses or NMLE. Washout is the most ordinary kinetic pattern of these tumors. In general, the imaging characteristics did not vary significantly with the proportion of the lobular component.

Keywords: Breast carcinoma; Lobular; Ductal; Mammography; Ultrasonography; Magnetic resonance imaging

\section{Introduction}

Breast cancer is a heterogeneous group of tumors with multivariate morphology, growth pattern, molecular profiles and response to treatment. The majority of invasive breast cancers $(72-80 \%)$ are categorized as invasive ductal carcinoma (IDC). The prevalence of the second most common type of breast cancer, invasive lobular

\footnotetext{
* Correspondence: G.DeLimaGomesdeMenezes@umcutrecht.nl 'Department of Imaging, University Medical Centre Utrecht, E01.132, P.O. Box 85500, 3508, GA Utrecht, The Netherlands

Full list of author information is available at the end of the article
}

carcinoma (ILC), accounts for 5 to 15\% (Biglia et al. 2007; Li et al. 2003; Verkooijen et al. 2003).

There is an extensive literature on clinical and imaging characteristics of both IDC and ILC (Acs et al. 2001; Arps et al. 2013; Brem et al. 2009; Kim et al. 2011; Korhonen et al. 2004; Lopez \& Bassett 2009; Sastre-Garau et al. 1996; Winston et al. 2000; Yang et al. 2007). Multiple differences in demographic and tumor features between these two histological types have been reported. Patients with ILC are generally older at the time of the diagnosis, (Sastre-Garau et al. 1996; Moran et al. 2009) ILC is usually larger in diameter, (Arpino et al. 2004; Biglia et al. 2013) is more 
frequently hormone receptor positive, (Arps et al. 2013; Arpino et al. 2004) has lower grade than IDC, (Arps et al. 2013; Arpino et al. 2004; Biglia et al. 2013) is more frequently multifocal, multicentric and bilateral, and the organ distribution of metastatic disease tends to spread to pelvic organs, gastrointestinal tract and also to distinct sites such as retroperitoneum, meninges, ovary and serosa.

ILC has the histological characteristic to spread in rows of single cell layers around normal ducts like a "spider web", infiltrating the preexisting stroma without inducing a strong desmoplastic response (Michael et al. 2008; Qureshi et al. 2006). This growth pattern causes minimum disruption of the normal anatomical structures than IDC, turning the radiological and clinical diagnostic of this tumor into a real challenge (Yeatman et al. 1995). This insidiously invasive nature makes the full extent of these tumors difficult to diagnose in screening. Mammogram may only reveal subtle changes or can even be completely normal. Mammographic sensitivity for detection of ILC varies between 57-92\% (Butler et al. 1999; Hilleren et al. 1991a; Le et al. 1992) and ILC has higher false negative rates than IDC (19 vs. 10\%) (Framarino Dei et al. 1995), making ILC more difficult to diagnose, especially in early stage. Ultrasound (US) is slightly more sensitive than mammography (sensitivity between 6895\%) and has shown to be more accurate in determine the (pathologic) size of the lesion, and also in identifying multifocality and multicentricity (Butler et al. 1999; Berg et al. 2004; Chapellier et al. 2000; Paramagul et al. 1995; Selinko et al. 2004). Magnetic resonance imaging (MRI) has become mainstream for diagnosis and work-up of breast cancer patients and many studies have demonstrated this imaging modality to have sensitivity above 90\% (Butler et al. 1999; Berg et al. 2004; Chapellier et al. 2000; Boetes et al. 1995; Boetes et al. 1997; Harms et al. 1993; Mumtaz et al. 1997; Orel et al. 1994; Orel \& Schnall 2001; Peters et al. 2008; Qayyum et al. 2002; Rodenko et al. 1996). MRI plays a fundamental role in providing additional information not obtained by conventional digital mammography and ultrasound, being of great importance in recognition of ipsilateral and contralateral lesions (Rodenko et al. 1996; Mann et al. 2008). The MONET trial demonstrated that breast MRI was associated with an increased re-excision rate and is not advised to be used routinely for preoperative work-up of patients with non-palpable breast cancer (Peters et al. 2011). However, various authors proposed preoperative breast MRI to have significant impact in treatment of patients with ILC (Kim et al. 2011; Lopez \& Bassett 2009; Michael et al. 2008; Qayyum et al. 2002; Mann et al. 2008; Boetes et al. 2004; Schelfout 2004). MRI has a superior accuracy (Berg et al. 2004; Boetes et al. 2004) in defining the extent of ILC and is, therefore, essential for a correct surgical planning and further treatment of these patients (Boetes et al. 1995; Orel et al. 1994; Rodenko et al. 1996; Mann et al. 2008; Peters et al. 2011; Lesser et al. 1982).
Invasive ductolobular carcinoma, also called invasive ductal carcinoma with lobular features (IDC-L), is intermediate in the histological spectrum from ILC to IDC, but the clinical and radiological presentation and behavior of this histological type have not been widely studied. It is therefore not well known if imaging features, clinicopathologic behavior, and outcome of these tumors are more comparable to IDC or to ILC.

The aim of this study was to therefore describe the spectrum of mammographic, sonographic and MRI features according to the BI-RADS ${ }^{\bullet}$ lexicon in patients with histologically proven invasive ductolobular carcinoma of the breast and to evaluate the relationship between the proportion of the lobular component and the imaging characteristics of these breast tumors.

\section{Methods}

\section{Patients}

Patients diagnosed with invasive breast carcinoma containing lobular features at the UMC Utrecht (The Netherlands) between January 2008 and October 2012 were considered. Only patients who underwent preoperative MRI, mammography and US were included in this study.

\section{Imaging acquisition}

For the mammograms, the standard craniocaudal view and mediolateral oblique were obtained using the Hologic Lorad Selenia full field digital mammography system. Additional views or spot compression were obtained when necessary. The US images were acquired using a Philips HD-11 XE digital imaging system (5-12 MHz linear probe).

The MRI scans were acquired with the patient in the prone position on 3 Tesla clinical MRI scanners (Achieva, Phillips Healthcare, Best, The Netherlands) equipped with dedicated phased-array bilateral breast coils (SENSE-Breast7TX and SENSE-Breast-4 MRI devices).

MRI imaging was performed according to our standard staging breast imaging protocol, which included a transverse high-resolution T1-weighted isotropic volume examination (THRIVE) [TE/TR $1.87 / 4.9 \mathrm{~ms}$; flip angle $10^{\circ}$; FOV $360 \times 360 \times 180 \mathrm{~mm}^{3}$, acquired voxel size $0.65 \times 0.65 \times$ $2.0 \mathrm{~mm}^{3}$, reconstructed voxel size $0.64 \times 0.64 \times 1.00 \mathrm{~mm}^{3}$ ) and a transverse SPAIR T2-weighted series (TE/TR 100/ $5508 \mathrm{~ms}$; inversion delay SPAIR $305 \mathrm{~ms}$; flip angle $90^{\circ}$; FOV $360 \times 360 \times 180 \mathrm{~mm}^{3}$, acquired voxel size $1.00 \cdot 1.46$. $2.0 \mathrm{~mm}^{3}$, reconstructed voxel size $0.64 \cdot 0.64 \cdot 2.00 \mathrm{~mm}^{3}$ ). The dynamic series consisted of contrast-enhanced fatsuppressed T1-weighted gradient echo images (TE/TR $1.24 / 3.3 \mathrm{~ms}$; flip angle $10^{\circ}$; FOV $360.360 \times 180 \mathrm{~mm}^{3}$, acquired voxel size $1.00 \cdot 1.00 \cdot 2.00 \mathrm{~mm}^{3}$, reconstructed voxel size $0.94 \cdot 0.94 \cdot 1.00 \mathrm{~mm}^{3}$; dynamic scan duration 
68 seconds). Images were acquired before and at 0, 69, 138, 206, 274 and 342 seconds after the administration of $0.1 \mathrm{mmol} / \mathrm{kg}$ Gadolinium-DTPA (Magnevist, Schering, Germany). The acquisition time of this scan package was approximately 25 minutes.

\section{Image interpretation}

Mammograms and US images were retrieved from the local PACS system and analyzed at a Sectra Workstation IDS7 (Sectra Imtec AB, Sweden). MRI examinations were processed by CADstream (Confirma, Inc., Kirkland, WA, USA). The images were interpreted by two dedicated breast radiologists. In addition, all images were reviewed and interpreted by a third radiologist, who was blinded to the proportion of the lobular component of each patient. In case of discordance with the original reports, a consensus was reached with a fourth dedicated breast radiologist with more than 20 years of experience in breast imaging.

All images were interpreted according to the guidelines of the BI-RADS ${ }^{\circ}$ lexicon (D’Orsi \& Dea 2003). Lesions were essentially divided into mass and non-masslike lesions in order to perform morphological analysis. Based on the BI-RADS ${ }^{\ominus}$ lexicon, (D'Orsi \& Dea 2003) lesions which had a mass as the main characteristic [isolated mass or dominant mass surrounded by smaller masses or foci of non-mass like enhancement (NMLE)] were defined as a mass-like lesion. Architectural distortion and NMLE (focal area, linear, ductal, segmental, regional, multiple regions, diffuse enhancement, and multiple enhancing foci) were defined as descriptors of non-mass-like lesion. Time intensity curves were classified according to their pattern of initial rise (slow, medium, rapid) and according to the delayed phase (persistent, plateau, washout). Finally, each lesion was scored according to the BI-RADS ${ }^{\bullet}$ lexicon; (D’Orsi \& Dea 2003) 0 - Finding for which additional evaluation is needed, 1 - No abnormal enhancement, no lesion found, 2 - Benign finding, 3 - Probably benign finding, (short interval follow-up), 4 - Suspicious abnormality, 5 - Highly suggestive of malignancy, 6 - Known cancer biopsy-proven malignancy diagnosis on the imaged finding prior to definitive therapy.

Tumor extent and additional disease were defined as follows:

- Multifocality: an additional malignant lesion in the same quadrant, separated from the index tumor by benign tissue.

- Multicentricity: an additional malignant lesion in a different quadrant than the index cancer.

- Contralateral disease: an additional malignant lesion found in contralateral breast.
- Multiplicity: two or more of these features: multifocality, multicentricity and contralateral disease.

Additional findings were considered true positives when histopathological analysis of either preoperative work-up or surgical specimen has shown malignancy [invasive carcinoma or ductal carcinoma in situ (DCIS)].

\section{Histological analysis}

All slices of ductolobular carcinoma $(n=113)$ were reviewed by a dedicated breast radiologist to quantify the lobular component, defined as a proportion of the invasive cancer.

\section{Statistical analysis}

Data were analysed using SPSS 17.0 software (SPSS, Inc., Chicago, IL, USA). Chi - square tests were used to compare proportions of the lobular component to the imaging characteristics and to compare the proportions of lobular component to the additional findings in our sample. For statistical purpose, proportion of the lobular component was grouped into three different categories: $\leq 20 \%, 21-$ $94 \%$, and $\geq 95 \%$. Results were considered significant at $p<0.05$.

\section{Results}

Between January 2008 and October 2012, 505 patients were diagnosed with breast cancer and invasive ductal carcinoma with lobular features was reported in 30\% $(155 / 505)$ of the patients. Of these 155, 41 patients were excluded due to technical problems in performing MRI, obesity, claustrophobia, impossibility of obtaining mammography or ultrasound before MRI and personal reasons. The remaining 113 patients who underwent mammography, ultrasound and MRI were selected for this study. The age at diagnosis of the 113 patients ranged from 34 to 87 years with a mean of 57.4 years.

There were 41 patients with a proportion of $\leq 20 \%$ of lobular component, 36 patients with a proportion of 21 to $94 \%$, and 36 patients with $\geq 95 \%$ of lobular component.

\section{Mammographic findings}

Mammographic findings are presented in Table 1. A mass was the most common mammographic finding, observed in $54.8 \%$ of cases. In $46.0 \%$ of cases, we found an isolated mass and, in $8.8 \%$, a mass was found associated with microcalcifications. Isolated microcalcifications were seen in $11.5 \%$ of the patients. Architectural distortion was found in $10.6 \%$ of cases and asymmetries (associated or not with microcalcifications) were noticed in $8.1 \%$ of cases. Normal findings were observed in $15.0 \%$ of the patients. 
Table 1 Mammographic findings of invasive ductolobular carcinomas of the breast

\begin{tabular}{|c|c|}
\hline Findings & $n=113$ \\
\hline Benign/Normal & $15.0 \%(17 / 113)$ \\
\hline Mass & $46.0 \%(52 / 113)$ \\
\hline Mass with calcification & $8.8 \%(10 / 113)$ \\
\hline Calcification only & $11.5 \%(13 / 113)$ \\
\hline Architectural distortion & $10.6 \%(12 / 113)$ \\
\hline Focal asymmetry or asymmetry & $7.1 \%(8 / 113)$ \\
\hline Asymmetry and calcification & $1.0 \%(1 / 113)$ \\
\hline Mass shape & $n=62$ \\
\hline Round/Oval & $8.1 \%(5 / 62)$ \\
\hline Lobular & $3.2 \%(2 / 62)$ \\
\hline Irregular & $88.7 \%(55 / 62)$ \\
\hline Mass Margin & $n=62$ \\
\hline Circunscribed & $4.8 \%(3 / 62)$ \\
\hline Not Circunscribed & $24.2 \%(15 / 62)$ \\
\hline Spiculated & $71.0 \%(44 / 62)$ \\
\hline Mass Density & $n=62$ \\
\hline Isodense & $88.7 \%(55 / 62)$ \\
\hline Hyperdense & $11.3 \%(7 / 62)$ \\
\hline Associated Findings & $n=113$ \\
\hline Nipple retraction & $3.5 \%(4 / 113)$ \\
\hline Skin Thickening & $10.6 \%(12 / 113)$ \\
\hline Enlarged axillary lymph nodes & $3.5 \%(4 / 113)$ \\
\hline
\end{tabular}

Prevalence of normal findings was higher (25.0\%) in patients with $\geq 95 \%$ of lobular component vs. $12.2 \%$ in the $\leq 20 \%$ lobular component group (Table 2). However, this difference had no statistical significance $(p=0.115)$.

Considering only mass lesions $(n=62)$, a total of $62.9 \%$ were simultaneously irregular, spiculated and isodense to the fibroglandular parenchyma (Figure 1a). Isodense mass was more frequently associated with smaller lobular component $(p=0.016)$. Circumscribed masses were seen in $2.6 \%(3 / 113)$ of the patients.

Table 2 Imaging findings of invasive ductolobular carcinomas of the breast according to proportion of the lobular component

\begin{tabular}{lccc}
\hline & \multicolumn{3}{c}{ Proportion lobular component } \\
\hline Findings & $\leq 20 \%$ & $21-94 \%$ & $\geq 95 \%$ \\
& $(\mathrm{n}=41)$ & $(\mathrm{n}=36)$ & $(\mathrm{n}=36)$ \\
Normal Mammographic Findings & $12.2 \%(5)$ & $8.3 \%(3)$ & $25.0 \%(9)$ \\
Multifocality & $21.9 \%(9)$ & $27.7 \%(10)$ & $33.3 \%(12)$ \\
Multicentricity & $9.7 \%(4)$ & $19.4 \%(7)$ & $25.0 \%(9)$ \\
Contralateral Disease & $9.7 \%(4)$ & $11.1 \%(4)$ & $16.6 \%(6)$ \\
Multiplicity & $7.3 \%(3)$ & $13.8 \%(5)$ & $30.5 \%(11)$ \\
\hline
\end{tabular}

The prevalence of other mammographic lesions (microcalcifications, architectural distortion, asymmetries), mass shape and mass margins did not vary significantly according to the proportion of the lobular component.

Additional findings (nipple retraction and skin thickening) were seen in $14.1 \%(16 / 113)$ of the patients (Figures 2a and $3 a)$.

\section{Ultrasound findings}

Ultrasound findings are summarized in Table 3. Of the 113 cancers, 109 (96.5\%) lesions were masses localized in the breast, $3(2.6 \%)$ patients had normal exams and 1 (0.9\%) patient had a parasternal mass. These masses $(\mathrm{n}=109)$ found in our study were mainly irregular (92.7\%), spiculated (60.6\%), hypoechoic (91.8\%), with posterior acoustic shadowing (64.2\%). These four characteristics were observed simultaneously in $46.8 \%$ of the patients (Figures $1 \mathrm{~b}$ and $4 \mathrm{~b}$ ). Microlobulated margin was seen in $19.3 \%$ of the patients and absence of posterior acoustic features was found in $30.3 \%$ of cases.

Taking into account the lobular component, angular margins were more prevalent in patients with a bigger lobular component, with $11.1 \%$ in the $\geq 95 \%$ group and $4.8 \%$ in the $\leq 20 \%$ group. However, these results had no statistical significance. Findings concerning to mass shape, echogenicity and posterior acoustic features had similar prevalence in all groups.

\section{MRI findings}

Of all tumors $(\mathrm{n}=113), 89.4 \%(101)$ were classified as "mass-like" lesion (see Table 4). From these 101, 56.4\% were isolated masses, $37.6 \%$ were a dominant mass associated with NMLE features and 6.0\% were dominant masses surrounded by smaller masses. The most common findings for mass lesions were low signal on $\mathrm{T} 1$ in $99.0 \%$ of cases and moderate signal in $43.6 \%$ of cases on T2-weighted images. Concerning mass shape, the masses found were predominately irregular (86.1\%). Regarding the margins of these masses, spiculated or irregular margins were found in $91.1 \%$. Heterogeneous enhancement pattern was seen in $59.4 \%$ of cases.

$39.8 \%$ of patients presented with NMLE features and they could be found isolated or associated with other lesions (adjacent to a dominant mass or to an architectural distortion). Considering all cases with NMLE features found in our sample (45 in total), $71.1 \%$ presented as focal areas. The internal enhancement was homogeneous in $69.0 \%$ and all cases were asymmetric.

Lesions with isolated NLME aspect were seen in 5.3\% $(6 / 113)$ of our patients. Architectural distortion and architectural distortion associated with NMLE were seen in $2.6 \%$ and $0.9 \%$ of the patients, respectively. Normal exams were found in 2 patients (1.8\%). 

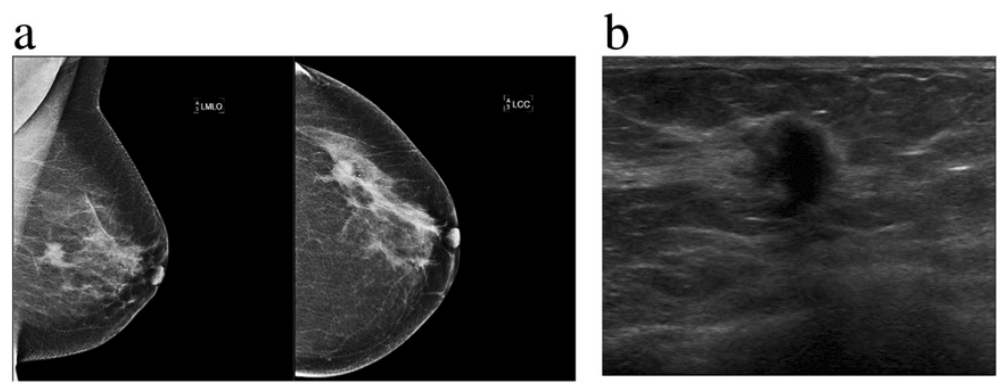

Figure 148 year old woman, with positive family history for breast cancer, presented with a palpable lump on the left breast, finally diagnosed as ductolobular carcinoma with a $\leq \mathbf{2 0} \%$ proportion of the lobular component. a) Mediolateral oblique and craniocaudal mammograms show an irregular and spiculated mass. A comet-tail like projection arises from the anterior and posterior margins and a discrete retraction around the lesion can be seen. b) Ultrasound of the left breast demonstrates an irregular, hypoechoic and vertically oriented mass, with echogenic halo at 3 o'clock position.

Considering all lesions with available kinetic data $(\mathrm{n}=103)$, rapid initial rise was seen in $86.4 \%$ of cases and in $61.2 \%$ of cases had washout pattern in delayed phase. In $7.1 \%$ of cases, kinetic data was not available and $6.8 \%$ of the patients showed benign pattern (persistent curve) in delayed phase. In kinetic delayed phase, "plateau" curve was more frequently observed in cases with bigger lobular component (36\% in both the 21 $94 \%$ and $\geq 95 \%$ groups vs. $17 \%$ in the $\leq 20 \%$ group) (Figures 3c, d and 5c). Washout was more prevalent in tumors with a smaller lobular component (63.4\% in the $\leq$ $20 \%$ group vs. $50.0 \%$ in the $\geq 95 \%$ group) (Figure 2 e) . Despite these differences, there was no statistical relevant difference among those groups $(p \geq 0.05)$. Mass shape, mass margins, patterns of mass enhancement and NMLE characteristics did not show statistical significant variations according to the proportion of the lobular component.

We found 46 associated findings in 36 patients (31.8\%), such as nipple retraction, skin thickening (focal or diffuse), edema, hematoma/blood and pre-contrast high ductal signal. Invasion of the pectoral muscle, confirmed by histopathological analysis, was found in 5 (4.4\%) patients, nipple invasion in $3(2.6 \%)$ patients and skin invasion in $2(1.8 \%)$ cases. Chest wall invasion was seen in $1(0.9 \%)$ patient, associated to pectoral muscle ingrowth and, in $2(1.8 \%)$ cases, nipple, skin and pectoral muscle ingrowth were found together (Figures 2b, c, d, $3 \mathrm{~b}$ and $5 \mathrm{a})$.

\section{Lymphadenopathy}

Lymph node metastasis was histologically reported in 57 patients. From these, $38.5 \%$ were seen by imaging. In 5 cases, lymphadenopathy was seen both in MRI and US and in 3 cases it was seen in MRI, US and mammography. Prevalence of lymphadenopathy did not show significant differences according to proportion of the lobular component.

\section{Additional findings}

Multifocality and Multicentricity were found in 31 (27.4\%) and $20(17.7 \%)$ patients respectively. Multiplicity was found in 19 cases (16.8\%) and contralateral disease was seen in $14(12.3 \%)$ patients (Figure $2 \mathrm{c}$ and $4 \mathrm{~b})$. Taking into account the proportion of lobular component, contralateral disease, multifocality and multicentricity had higher prevalence rates in patients with bigger lobular component (Table 2), but there was no statistical significance. Nevertheless, multiplicity was more likely to be found in patients with a bigger lobular component $(p=0.021)$.

\section{Size}

At mammography and US, tumor size ranged from 4 to $6.0 \mathrm{~cm}$ (mean, $1.78 \mathrm{~cm}$ ) and on MRI tumor size ranged from 2 to $9.4 \mathrm{~cm}$ (mean, $2.50 \mathrm{~cm}$ ). The sizes found on pathology ranged from 2 to $12.0 \mathrm{~cm}$ (mean, $2.9 \mathrm{~cm}$ ).

\section{Discussion \\ Mammography}

The characteristics of IDC and ILC have been extensively described in literature. The dispersed infiltrating growth pattern of ILC with very little desmoplastic reaction and, consequently, the development of palpable lesion or tumors detectable in imaging exams, is less frequent. Normal or benign findings are more common in ILC than in IDC ( $8 \%-16 \%$ vs. $1.1 \%)$ (Li et al. 2003; Kim et al. 2011; Selinko et al. 2004; Mann et al. 2008) and false negative rates for ILC in mammography range from 14 to $19 \%$ (Kim et al. 2011; Hilleren et al. 1991b; Krecke \& Gisvold 1993).

In the current study, we observed 15\% (17/113) of normal or benign mammograms, which might demonstrate a similar behavior to ILC. It was also interesting that patients with tumors with $\geq 95 \%$ of lobular component had a higher prevalence of normal findings in 

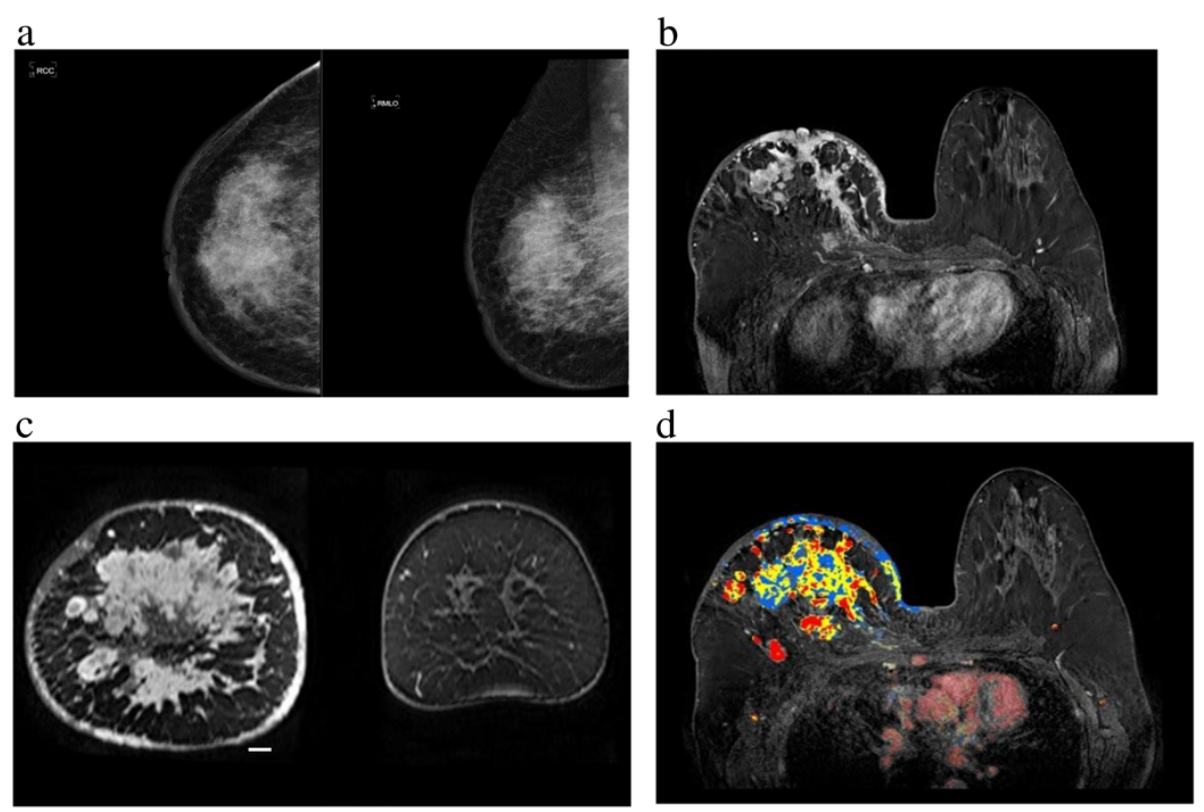

e

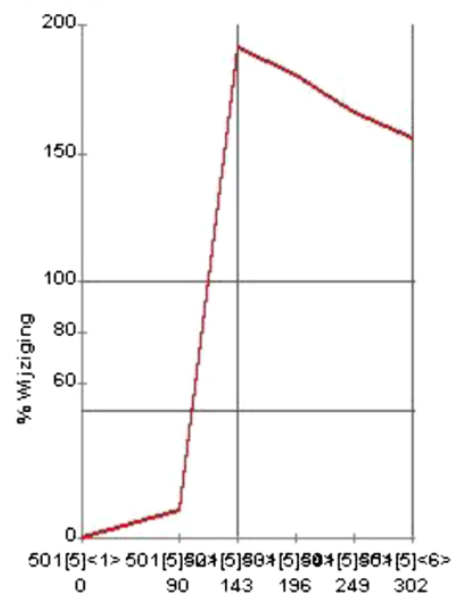

Figure 259 year-old patient with mastitis carcinomatosa (inflammatory carcinoma), finally diagnosed as ductolobular carcinoma with $a \geq 95 \%$ proportion of the lobular component. a) Craniocaudal and mediolateral mammograms show extensive skin and trabecular thickening/coarsening of the right breast and diffusely increased breast density, associated with multiple calcifications in the upper outer breast. b) MRI shows multiple confluent masses, with irregular margins and heterogeneous internal enhancement pattern. Skin thickening and invasion of the nipple and the pectoral muscle are also observed. These are the typical features of inflammatory carcinoma. Multicentricity (multifocal and multicentric disease simultaneously) can also be observed in coronal projection (c). $\mathbf{d}$ and e) Post-processed color parametric map image demonstrates multiple areas of malignant enhancement in axial projection. Kinetic curve demonstrates typical malignant pattern (rapid initial rise and washout).

mammography than patients with $\leq 20 \%$ of lobular component ( 25 vs. $12 \%$, respectively), but these differences were not statistically significant.

ILC is usually seen as a mass (44\%-65\% of cases), (Lopez \& Bassett 2009; Helvie et al. 1993) having predominantly irregular and spiculated margins (63-71\%) (Helvie et al. 1993; Evans et al. 2002) and is usually isodense when compared to the fibroglandular tissue (Kim et al. 2011; Lopez \& Bassett 2009; Sickles 1991). These numbers were similar to our present findings. Mass was found in $54.8 \%$ of our sample and $62.9 \%$ of these masses were simultaneously irregular, spiculated and isodense.

ILC spreading diffusely through the breast stroma leads to lower tendency to form round and circumscribed masses, only seen $1 \%-3 \%$ of cases of ILC (Le et al. 1992). The lobular component of ductolobular tumors might lead to a similar behavior and, in our study, circumscribed masses were indeed only found in $2.6 \%$ of our cases.

Architectural distortion was seen in $10.6 \%$ of cases and asymmetries were found in $7.9 \%$ of cases. Literature 


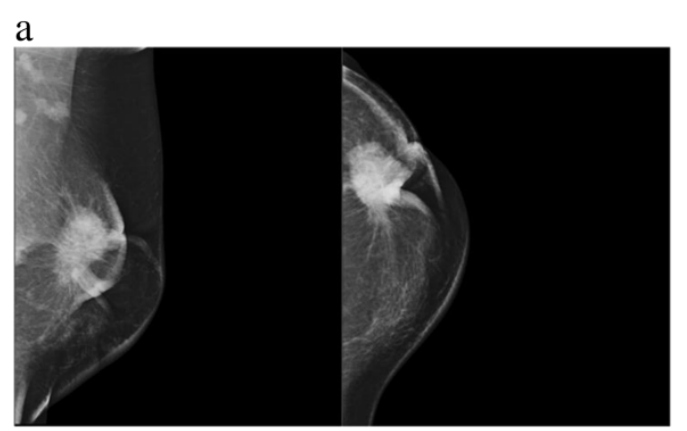

C

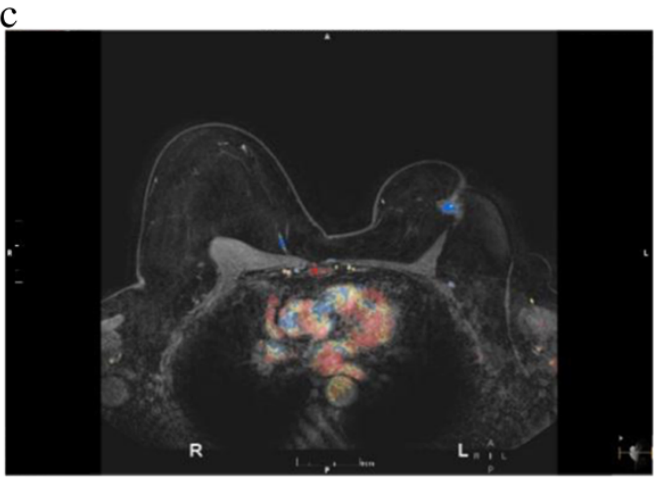

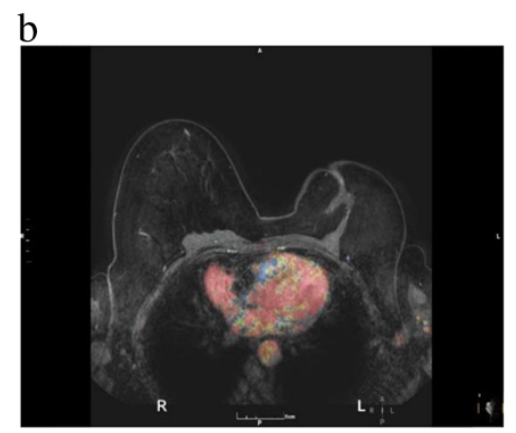

d

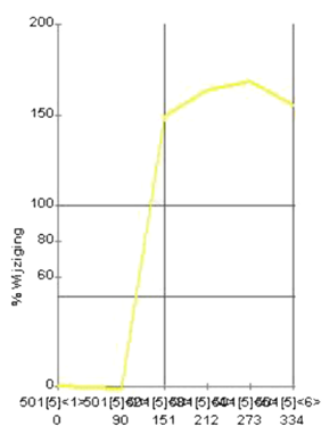

Figure 352 year-old woman with nipple retraction and metastasis to the right eye, finally diagnosed as ductolobular carcinoma with a 21 - 94\% proportion of the lobular component. a) Left mediolateral oblique and craniocaudal mammograms show a star shaped mass in center of the breast, associated with calcifications, skin thickening and nipple retraction. b) Axial contrast material-enhanced with fat suppression show a spiculated mass. There is nipple retraction, skin thickening and pectoral muscle invasion. c and $\mathbf{d}$ ) Post-processed color parametric map and kinetic curve predominantly demonstrate persistent enhancement and a small central area of plateau.

findings refers $10 \%-16 \%$ of ILC cases manifesting as architectural distortion (Hilleren et al. 1991b; Helvie et al. 1993) and $4 \%-13 \%$ of cases expressing as asymmetries (Hilleren et al. 1991b; Helvie et al. 1993). Our findings are comparable to these ILC mammographic lesions. However, architectural distortion is the second most common finding in ILC (Hilleren et al. 1991b; Helvie et al. 1993) and architectural distortion was the third most common radiological abnormality in our study.

It is well known that microcalcifications are much less common when comparing ILC and other breast carcinomas (4-24\% vs. 41\%) (Le et al. 1992). The prevalence of microcalcifications in our study was similar to the referred prevalence of these findings in ILC (11.5\%).

\section{Ultrasonography}

US is considered more sensitive than mammography in detecting ILC. Literature reports sensitivities ranging from 68 to 98\% (Paramagul et al. 1995; Selinko et al. 2004) and this imaging modality is also more accurate in identifying multifocality, multicentricity and size of the lesion (Selinko et al. 2004). According to Kim and Butler, mass has been described as being the most common lesion found in US in cases of ILC (60.5 - 100\%) and both authors agreed that an irregular, hypoechoic mass, with spiculated margins and posterior acoustic shadowing is the most ordinary pattern seen in US images of ILC (Kim et al. 2011; Butler et al. 1999). Our results are consistent with these numbers. However, Kim et al. described US features of ILC and IDC as being very similar, except for posterior acoustic features, which has been described as being more related to ILC (Kim et al. 2011).

\section{MR imaging}

MRI has proven to have a high overall sensitivity (approximately 95\%) (Mann et al. 2008; Kneeshaw 2003) and, in adjunct to mammography and US, has an essential importance in diagnostic and staging of ILC. MRI has a moderate specificity (67.4\%) (Bluemke et al. 2004) and the routinely clinical use of this imaging modality might lead to unnecessary procedures (Peters et al. 2011). However, in ILC cases, MRI is superior to other imaging modalities in estimating tumor size, detecting multifocality, multicentricity, contralateral disease (Boetes et al. 1995; Orel et al. 1994; Rodenko et al. 1996; Mann et al. 2008; Peters et al. 2011; Lesser et al. 1982). and also affecting surgical management in $28 \%$ of cases (Mann et al. 2008; Weinstein 2001). Mass is considered the most common manifestation of ILC at MRI and the 
Table 3 Ultrasonographic findings of invasive ductolobular carcinomas of the breast

\begin{tabular}{ll}
\hline Findings & $\mathbf{n}=\mathbf{1 1 3}$ \\
\hline Benign & $2.6 \%(3 / 113)$ \\
Mass & $96.5 \%(109 / 113)$ \\
Other findings (parasternal mass) & $0.9 \%(1 / 113)$ \\
Mass Shape & $\mathbf{n}=\mathbf{1 0 9}$ \\
Round/Oval & $7.3 \%(8 / 109)$ \\
Irregular & $92.7 \%(101 / 109)$ \\
Mass Margin & $\mathbf{n}=\mathbf{1 0 9}$ \\
Circumscribed & $1.8 \%(2 / 109)$ \\
Indistinct & $10.0 \%(11 / 109)$ \\
Angular & $8.3 \%(9 / 109)$ \\
Microlobulated & $19.3 \%(21 / 109)$ \\
Spiculated & $60.6 \%(66 / 109)$ \\
Mass Echogenicity & $\mathbf{n}=109$ \\
Complex echoic & $6.4 \%(7 / 109)$ \\
Hypoechoic & $91.8 \%(100 / 109)$ \\
Isoechoic & $1.8 \%(2 / 109)$ \\
Mass Posterior acoustic feature & $\mathbf{n}=\mathbf{1 0 9}$ \\
No feature & $30.3 \%(33 / 109)$ \\
Enhancement & $5.5 \%(6 / 109)$ \\
Shadowing & $64.2 \%(70 / 109)$ \\
Essociated Findings & $\mathbf{n}=\mathbf{1 1 3}$ \\
\hline & $8.8 \%(10 / 113)$ \\
\hline
\end{tabular}

incidence varies substantially (45\%-95\%) (Kim et al. 2011; Rodenko et al. 1996; Schelfout 2004; Weinstein 2001; Yeh et al. 2003). Most of these studies have not described ILC findings in MRI strictly according to the BI-RADS lexicon. However, Hye $\mathrm{Na}$ Jung and Kim found $92 \%$ and $88.8 \%$ of ILC cases presented as mass - like lesion according to the BI-RADS ${ }^{\circ}$ (Kim et al. 2011; Jung et al. 2013). These findings are consistent with our research $(89.4 \%$ of cases presenting as mass - like tumors).

In a literature review, Mann et all described $85.5 \%$ (65/76) of ILC tumors presenting as an irregular or spiculated mass (Mann et al. 2008). Our study had similar results and $91 \%(92 / 101)$ of the lesions were described as irregular or spiculated masses.

T1 and T2 features of lobular tumors are not frequently mentioned in literature. Levrini reported 95.2\% $(20 / 21)$ of cases of ILC tumors being hypo- and hyperintense lesions on T2 weighted TSE images (Levrini et al. 2008). Unfortunately all of our patients underwent diagnostic procedures within 10 days before MRI. The hemorrhage, edema and necrosis that result from these procedures may have changed $\mathrm{T} 1$ and/or T2 signal, which makes an accurate analysis more difficult.

Not many studies refer to the kinetic behavior of ILC. The infiltrative growth pattern of these tumors seems not to require extensive neovascularization and the lack of endothelial growth factor found in lobular tumors turns the new vessels to grow more slowly and having better maturation, resulting in less permeable capillaries (Lee et al. 1998). Some studies found ILC having delayed maximum enhancement and wash out pattern was not observed in the majority of tumors (Trecate et al. 2001; Sittek et al. 1998). These features might be due the histological behavior of ILC. Indeed, the prevalence of "plateau" curve in our study was higher in groups of patients with higher lobular component, and washout was more prevalent in groups of patients with lower lobular component. However, these differences were not statistically significant.

More recent studies refer $70.3 \%$ to $95.2 \%$ of ILC lesions having washout pattern (Kim et al. 2011; Levrini et al. 2008; Mann et al. 2011). Considering all available kinetic curves and not taking into account the proportion of the lobular
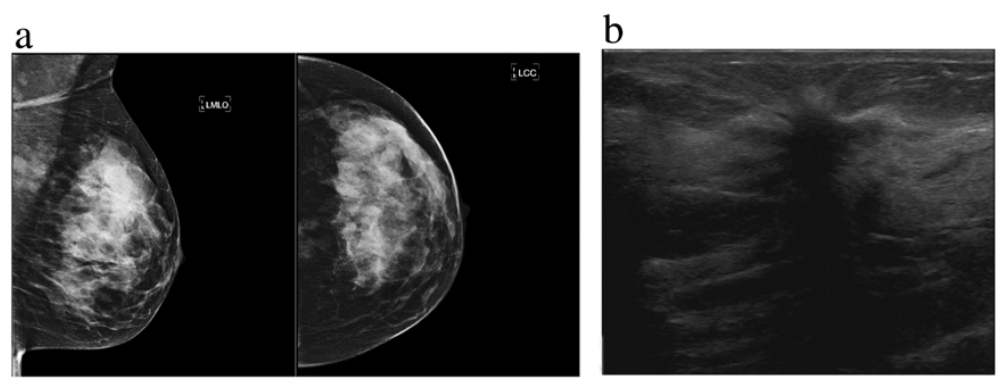

Figure 4 A 43 year old woman presenting with focal thickening in the left breast, finally diagnosed as ductolobular carcinoma with a $\leq \mathbf{2 0} \%$ proportion of the lobular component. a) Mediolateral and craniocaudal mammograms of the left breast show a spiculated mass in the upper outer quadrant. b) Ultrasound shows a hypoechoic and spiculated mass, with echogenic halo at and posterior acoustic shadowing (upper outer quadrant). 
Table 4 MRI findings of invasive ductolobular carcinomas of the breast according to the BI-RADS ${ }^{\oplus}$ (NMLE = non-mass like enhancement)

\begin{tabular}{l} 
Lesion Type \\
\hline Normal \\
Mass $^{\mathrm{a}}$ \\
Mass + NMLE \\
NMLE \\
Architectural distortion \\
Architectural distortion + NML \\
Mass T1 \\
Low \\
Mod \\
High \\
High central low peripheric
\end{tabular}

Mass T2

Low

Mod

High

Low central high peripheric

Mass Shape
Round/Oval
Lobular
Irregular
Mass Margin
Smooth
Irregular
Spiculated
Mass Enhancement
Homogeneous
Heterogeneous
Rim enhancement
Dark internal septation
Enhancing internal septation
Central enhancement
NMLE Distribution
Focal area
Linear
Ductal
Segmental
Regional
Multiple Regions
Diffuse

NMLE Internal Enhancement

Homogeneous

Heterogeneous

$\mathrm{n}=\mathbf{1 1 3}$

$1.8 \%(2 / 113)$

$55.8 \%(63 / 113)$

$33.6 \%(38 / 113)$

$5.3 \%(6 / 113)$

$2.6 \%(3 / 113)$

$0.9 \%(1 / 113)$

$\mathrm{n}=101$

99.0\% (100/101)

$0 \%(0 / 101)$

$0 \%(0 / 101)$

$1.0 \%(1 / 101)$

$\mathrm{n}=101$

7.9\% (8/101)

$43.6 \%(44 / 101)$

$37.6 \%(38 / 101)$

$10.9 \%(11 / 101)$

$\mathrm{n}=101$

$6.0 \%(6 / 101)$

$7.9 \%(8 / 101)$

$86.1 \%(87 / 101)$

$\mathrm{n}=101$

8.9\% (9/101)

$36.6 \%(37 / 101)$

$54.5 \%(55 / 101)$

$\mathrm{n}=101$

$15.8 \%(16 / 101)$

$59.4 \%(60 / 101)$

$24.8 \%(25 / 101)$

$0 \%(0 / 101)$

$0 \%(0 / 101)$

$0 \%(0 / 101)$

$\mathrm{n}=\mathbf{4 5}$

$71.1 \%(32 / 45)$

$11.1 \%(5 / 45)$

$4.5 \%(2 / 45)$

$2.2 \%(1 / 45)$

$2.2 \%(1 / 45)$

$6.7 \%(3 / 45)$

$2.2 \%(1 / 45)$

$\mathrm{n}=\mathbf{4 5}$

$69.0 \%(31 / 45)$

$22.2 \%(10 / 45)$
Table 4 MRI findings of invasive ductolobular carcinomas of the breast according to the BI-RADS $^{\oplus}$ (NMLE $=$ non-mass like enhancement) (Continued)

\begin{tabular}{ll}
\hline Stippled, punctate & $0 \%(0 / 45)$ \\
Clumped & $4.4 \%(2 / 45)$ \\
Reticular, dendritic & $0 \%(0 / 45)$ \\
Rim enhancement & $4.4 \%(2 / 45)$ \\
NMLE Symmetry & $\mathbf{n}=\mathbf{4 5}$ \\
Symmetric & $0 \%(0 / 45)$ \\
Asymmetric & $100.0 \%(45 / 45)$ \\
Kinetic Pattern Initial Rise $_{\text {Not available }}$ & $\mathbf{n}=\mathbf{1 1 3}$ \\
Slow $^{\text {b }}$ & $7.1 \%(8 / 113)$ \\
Medium $^{\mathrm{b}}$ & $0 \%(0 / 103)$ \\
Rapid $^{\mathrm{b}}$ & $13.6 \%(14 / 103)$ \\
Kinetic Pattern Delayed Phase & $86.4 \%(89 / 103)$ \\
Not available $_{\text {Persistent }}{ }^{\text {b }}$ & $\mathbf{n}=\mathbf{1 1 3}$ \\
Plateau $^{\mathrm{b}}$ & $7.1 \%(8 / 113)$ \\
Washout $^{\mathrm{b}}$ & $6.8 \%(7 / 103)$ \\
\hline
\end{tabular}

${ }^{a}$ Isolated mass or dominant mass surrounded by smaller masses.

${ }^{b}$ Excluding non-available and normal exams $(n=103)$.

component, washout was the most common pattern in our analysis (61.2\%), but still lower than the numbers referred from these authors. However, Mann at al. showed that when CAD-application was used to evaluate the kinetic curve of lesions of ILC and IDC, washout pattern has a very similar prevalence in both tumors, which is not the case for visual assessment. In this latter case, IDC has a much higher prevalence of washout than ILC (Mann et al. 2011). The use of CADstream software to obtain the kinetic curves in our study might be the explanation for the higher washout pattern prevalence.

\section{Lymphadenopathy}

Arps et al. described IDC-L as having a higher frequency of nodal metastasis when compared to IDC and ILC (51 vs. 34 and $45 \%$, respectively) (Arps et al. 2013). A similar result was seen in our study and lymph node metastasis was found in $50.4 \%$ of our sample (57/113), even though there was no statistical significance between prevalence of lymphadenopathy and proportions of lobular component.

\section{Additional findings}

Since comprehensive studies about mixed tumors are missing in literature, it is difficult to put our present results into perspective. Arps et al. compared clinicopathologic features and outcomes of 183 cases of IDC-L with lobular features with 1499 patients with IDC and 375 


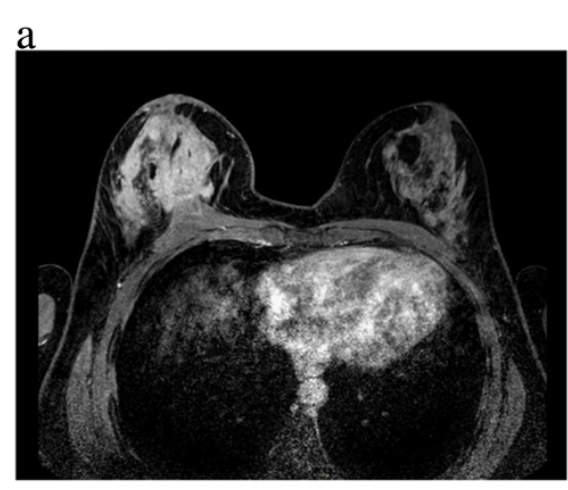

\section{b}
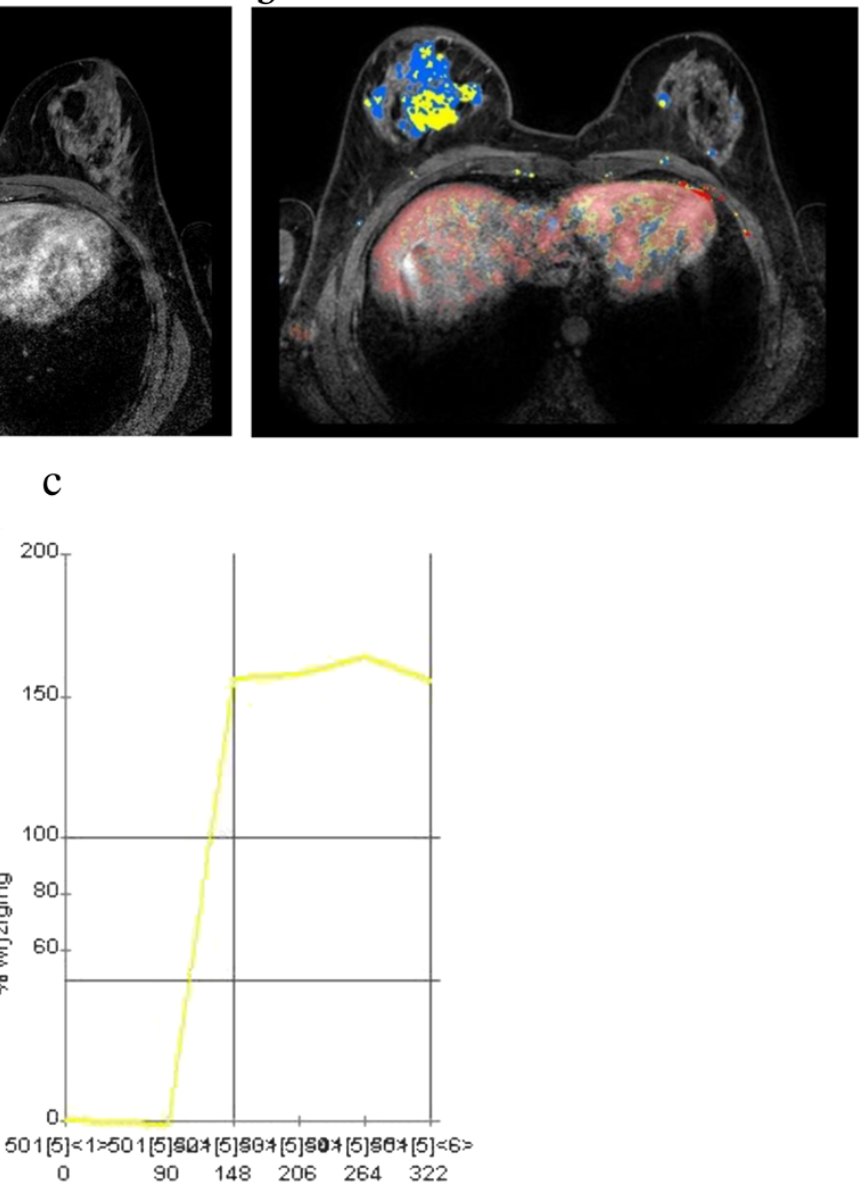

Figure 549 year-old patient with palpable thickening in the right breast, finally diagnosed as ductolobular carcinoma with a $\leq 20 \%$ proportion of the lobular component. a) Multiple irregular, confluent and heterogeneous masses in the right breast associated with skin thickening and pectoral muscle invasion. $\mathbf{b}$ and $\mathbf{c}$ ) Contralateral disease is better seen in color parametric map (axial projection). Observe the enhancement pattern (rapid initial rise and plateau) commonly observed in invasive lobular carcinomas.

patients with ILC. The authors concluded that the clinicopathologic features and outcomes of IDC-L and ILC are very similar, irrespective of the proportion of the lobular component (Arps et al. 2013).

In our study, not only imaging characteristics did not vary significantly according to the lobular component, but also multifocality, multicentricity, contralateral disease and the proportions of lobular component did not show a statistically significant correlation.

However, the significant association between two or more of these additional findings (multiplicity) and bigger lobular component $(p=0.021)$ is in line with the higher rates of additional disease foci in patients with ILC (Boetes et al. 1995; Orel et al. 1994; Rodenko et al. 1996; Mann et al. 2008; Peters et al. 2011; Lesser et al. 1982).

\section{Conclusion}

To our knowledge, this is the first study to exclusively describe radiological features of invasive ductolobular carcinoma. They typically present as an irregular, spiculated and isodense mass at mammography, as a hypoechoic, irregular and spiculated mass with posterior acoustic shadowing on US, and as an isolated mass or as a dominant mass surrounded by smaller masses or NMLE on MRI. Washout is the most ordinary kinetic pattern of these tumors. Except for isodensity and multiplicity, the imaging characteristics did not vary significantly according to proportion of the lobular component. The imaging features and the high incidence of additional malignant imaging findings of invasive ductolobular carcinoma are therefore more similar to ILC than to IDC.

\section{Ethical standards}

The study complies with current Dutch legislation.

Competing interests

The authors declare that they have no competing interests. 


\section{Authors' contributions}

GLGM performed the statistical analysis under supervision of HMV. ELP and MAES selected patients for the study and provided histological information. PJVD provided patients information histological analyses and proportions of lobular component. GLGM, MAAJB, and RMP drafted the manuscript. All authors contributed to data analysis and interpretation. All authors read and approved the final manuscript.

\section{Author details}

'Department of Imaging, University Medical Centre Utrecht, E01.132, P.O. Box 85500, 3508, GA Utrecht, The Netherlands. 'Department of Surgery, University Medical Centre Utrecht, PO Box 85500, 3508, GA Utrecht, Netherlands. ${ }^{3}$ Department of Pathology, University Medical Centre Utrecht, PO Box 85500, 3508, GA Utrecht, Netherlands.

Received: 18 October 2013 Accepted: 21 October 2013 Published: 20 November 2013

\section{References}

Acs G, Lawton TJ, Rebbeck TR, LiVolsi VA, Zhang PJ (2001) Differential expression of E-cadherin in lobular and ductal neoplasms of the breast and its biologic and diagnostic implications. Am J Clin Pathol 115(1):85-98

Arpino G, Bardou VJ, Clark GM, Elledge RM (2004) Infiltrating lobular carcinoma of the breast: tumor characteristics and clinical outcome. Breast Cancer Res 6(3):R149-R156

Arps DP, Healy P, Zhao L, Kleer CG, Pang JC (2013) Invasive ductal carcinoma with lobular features: a comparison study to invasive ductal and invasive lobular carcinomas of the breast. Breast Cancer Res Treat 138(3):719-726

Berg WA, Gutierrez L, NessAiver MS, Carter WB, Bhargavan M, Lewis RS, loffe OB (2004) Diagnostic accuracy of mammography, clinical examination, US, and MR imaging in preoperative assessment of breast cancer. Radiology 233(3):830-849

Biglia N, Mariani L, Sgro L, Mininanni P, Moggio G, Sismondi P (2007) Increased incidence of lobular breast cancer in women treated with hormone replacement therapy: implications for diagnosis, surgical and medical treatment. Endocr Relat Cancer 14(3):549-567

Biglia N, Maggiorotto F, Liberale V, Bounous VE, Sgro LG, Pecchio S, D'Alonzo M, Ponzone R (2013) Clinical-pathologic features, long term-outcome and surgical treatment in a large series of patients with invasive lobular carcinoma (ILC) and invasive ductal carcinoma (IDC). Eur J Surg Oncol 39(5):455-460

Bluemke DA, Gatsonis CA, Chen MH et al (2004) Magnetic resonance imaging of the breast prior to biopsy. JAMA 292(22):2735-2742

Boetes C, Mus RD, Holland R, Barentsz JO, Strijk SP, Wobbes T, Hendriks JH, Ruys SH (1995) Breast tumors: comparative accuracy of MR imaging relative to mammography and US for demonstrating extent. Radiology 197(3):743-747

Boetes C, Strijk SP, Holland R, Barentsz JO, Van Der Sluis RF, Ruijs JH (1997) Falsenegative MR imaging of malignant breast tumors. Eur Radiol 7(8):1231-1234

Boetes C, Veltman J, van DL, Bult P, Wobbes T, Barentsz JO (2004) The role of MRI in invasive lobular carcinoma. Breast Cancer Res Treat 86(1):31-37

Brem RF, loffe M, Rapelyea JA, Yost KG, Weigert JM, Bertrand ML, Stern LH (2009) Invasive lobular carcinoma: detection with mammography, sonography, MRI, and breast-specific gamma imaging. AJR Am J Roentgenol 192(2):379-383

Butler RS, Venta LA, Wiley EL, Ellis RL, Dempsey PJ, Rubin E (1999) Sonographic evaluation of infiltrating lobular carcinoma. AJR Am J Roentgenol 172(2):325-330

Chapellier C, Balu-Maestro C, Bleuse A, Ettore F, Bruneton JN (2000) Ultrasonography of invasive lobular carcinoma of the breast: sonographic patterns and diagnostic value: report of 102 cases. Clin Imaging 24(6):333-336

D'Orsi CJ, Dea MEI (2003) Breast Imaging Reporting and Data System: ACR BIRADS - Breast Imaging Atlas. American College of Radiology, Reston,VA

Evans WP, Warren Burhenne LJ, Laurie L, O'Shaughnessy KF, Castellino RA (2002) Invasive lobular carcinoma of the breast: mammographic characteristics and computer-aided detection. Radiology 225(1):182-189

Framarino Dei MM, Fiorelli C, Bandiera AF, Veneziano M, Galati GM, Porfiri L (1995) Infiltrating lobular carcinoma of the breast (ILC). Diagnostic and therapeutic aspects. Eur J Gynaecol Oncol 16(1):36-39

Harms SE, Flamig DP, Hesley KL, Meiches MD, Jensen RA, Evans WP, Savino DA, Wells RV (1993) MR imaging of the breast with rotating delivery of excitation off resonance: clinical experience with pathologic correlation. Radiology 187(2):493-501
Helvie MA, Paramagul C, Oberman HA, Adler DD (1993) Invasive lobular carcinoma. Imaging features and clinical detection. Invest Radiol 28(3):202-207

Hilleren DJ, Andersson IT, Lindholm K, Linnell FS (1991a) Invasive lobular carcinoma: mammographic findings in a 10-year experience. Radiology 178(1):149-154

Hilleren DJ, Andersson IT, Lindholm K, Linnell FS (1991b) Invasive lobular carcinoma: mammographic findings in a 10-year experience. Radiology 178(1):149-154

Jung HN, Shin JH, Han BK, Ko EY, Cho EY (2013) Are the imaging features of the pleomorphic variant of invasive lobular carcinoma different from classic ILC of the breast? Breast 22(3):324-329

Kim SH, Cha ES, Park CS, Kang BJ, Whang IY, Lee AW, Song BJ, Park J (2011) Imaging features of invasive lobular carcinoma: comparison with invasive ductal carcinoma. Jpn J Radiol 29(7):475-482

Kneeshaw PJ (2003) Dynamic contrast enhanced magnetic resonance imaging aids the surgical management of invasive lobular breast cancer. Eur J Oncol 29(1):32-37

Korhonen T, Huhtala H, Holli K (2004) A comparison of the biological and clinical features of invasive lobular and ductal carcinomas of the breast. Breast Cancer Res Treat 85(1):23-29

Krecke KN, Gisvold JJ (1993) Invasive lobular carcinoma of the breast: mammographic findings and extent of disease at diagnosis in 184 patients. AJR Am J Roentgenol 161(5):957-960

Le GM, Ollivier L, Asselain B, Meunier M, Laurent M, Vielh P, Neuenschwander S (1992) Mammographic features of 455 invasive lobular carcinomas. Radiology 185(3):705-708

Lee AH, Dublin EA, Bobrow LG, Poulsom R (1998) Invasive lobular and invasive ductal carcinoma of the breast show distinct patterns of vascular endothelial growth factor expression and angiogenesis. J Pathol 185(4):394-401

Lesser ML, Rosen PP, Kinne DW (1982) Multicentricity and bilaterality in invasive breast carcinoma. Surgery 91(2):234-240

Levrini G, Mori CA, Vacondio R, Borasi G, Nicoli F (2008) MRI patterns of invasive lobular cancer: T1 and T2 features. Radiol Med 113(8):1110-1125

$\mathrm{Li} \mathrm{Cl}$, Anderson BO, Daling JR, Moe RE (2003) Trends in incidence rates of invasive lobular and ductal breast carcinoma. JAMA 289(11):1421-1424

Lopez JK, Bassett LW (2009) Invasive lobular carcinoma of the breast: spectrum of mammographic, US, and MR imaging findings. Radiographics 29(1):165-176

Mann RM, Hoogeveen YL, Blickman JG, Boetes C (2008) MRI compared to conventional diagnostic work-up in the detection and evaluation of invasive lobular carcinoma of the breast: a review of existing literature. Breast Cancer Res Treat 107(1):1-14

Mann RM, Veltman J, Huisman H, Boetes C (2011) Comparison of enhancement characteristics between invasive lobular carcinoma and invasive ductal carcinoma. J Magn Reson Imaging 34(2):293-300

Michael M, Garzoli E, Reiner CS (2008) Mammography, sonography and MRI for detection and characterization of invasive lobular carcinoma of the breast. Breast Dis 30:21-30

Moran MS, Yang Q, Haffty BG (2009) The Yale University experience of early-stage invasive lobular carcinoma (ILC) and invasive ductal carcinoma (IDC) treated with breast conservation treatment $(\mathrm{BCT})$ : analysis of clinical-pathologic features, long-term outcomes, and molecular expression of $\mathrm{COX}-2, \mathrm{BCl}-2$, and $\mathrm{p} 53$ as a function of histology. Breast J 15(6):571-578

Mumtaz H, Hall-Craggs MA, Davidson T, Walmsley K, Thurell W, Kissin MW, Taylor I (1997) Staging of symptomatic primary breast cancer with MR imaging. AJR Am J Roentgenol 169(2):417-424

Orel SG, Schnall MD (2001) MR imaging of the breast for the detection, diagnosis, and staging of breast cancer. Radiology 220(1):13-30

Orel SG, Schnall MD, LiVolsi VA, Troupin RH (1994) Suspicious breast lesions: MR imaging with radiologic-pathologic correlation. Radiology 190(2):485-493

Paramagul CP, Helvie MA, Adler DD (1995) Invasive lobular carcinoma: sonographic appearance and role of sonography in improving diagnostic sensitivity. Radiology 195(1):231-234

Peters NH, Borel Rinkes IH, Zuithoff NP, Mali WP, Moons KG, Peeters PH (2008) Meta-analysis of MR imaging in the diagnosis of breast lesions. Radiology 246(1):116-124

Peters NH, van ES, van den Bosch MA et al (2011) Preoperative MRI and surgica management in patients with nonpalpable breast cancer: the MO. Eur J Cancer 47(6):879-886

Qayyum A, Birdwell RL, Daniel BL, Nowels KW, Jeffrey SS, Agoston TA, Herfkens RJ (2002) MR imaging features of infiltrating lobular carcinoma of the breast: histopathologic correlation. AJR Am J Roentgenol 178(5):1227-1232 
Qureshi HS, Linden MD, Divine G, Raju UB (2006) E-cadherin status in breast cancer correlates with histologic type but does not correlate with established prognostic parameters. Am J Clin Pathol 125(3):377-385

Rodenko GN, Harms SE, Pruneda JM, Farrell RS Jr, Evans WP, Copit DS, Krakos PA, Flamig DP (1996) MR imaging in the management before surgery of lobular carcinoma of the breast: correlation with pathology. AJR Am J Roentgenol 167(6):1415-1419

Sastre-Garau X, Jouve M, Asselain B, Vincent-Salomon A, Beuzeboc P, Dorval T, Durand JC, Fourquet A, Pouillart P (1996) Infiltrating lobular carcinoma of the breast. Clinicopathologic analysis of 975 cases with reference to data on conservative therapy and metastatic patterns. Cancer 77(1):113-120

Schelfout K (2004) Preoperative breast MRI in patients with invasive lobular breast cancer. Eur Radiol 14(7):1209-1216

Selinko VL, Middleton LP, Dempsey PJ (2004) Role of sonography in diagnosing and staging invasive lobular carcinoma. J Clin Ultrasound 32(7):323-332

Sickles EA (1991) The subtle and atypical mammographic features of invasive lobular carcinoma. Radiology 178(1):25-26

Sittek H, Perlet C, Untch M, Kessler M, Reiser M (1998) Dynamic MRmammography in invasive lobular breast cancer. Rontgenpraxis 51(7):235-242

Trecate G, Tess JD, Vergnaghi D, Bergonzi S, Mariani G, Ferraris C, Musumeci R (2001) Lobular breast cancer: how useful is breast magnetic resonance imaging? Tumori 87(4):232-238

Verkooijen HM, Fioretta G, Vlastos G et al (2003) Important increase of invasive lobular breast cancer incidence in Geneva, Switzerland. Int J Cancer 104(6):778-781

Weinstein SP (2001) MR imaging of the breast in patients with invasive lobular carcinoma. AJR Am J Roentgenol 176(2):399-406

Winston CB, Hadar O, Teitcher JB, Caravelli JF, Sklarin NT, Panicek DM, Liberman L (2000) Metastatic lobular carcinoma of the breast: patterns of spread in the chest, abdomen, and pelvis on CT. AJR Am J Roentgenol 175(3):795-800

Yang WT, Hennessy B, Broglio K, Mills C, Sneige N, Davis WG, Valero V, Hunt KK, Gilcrease MZ (2007) Imaging differences in metaplastic and invasive ductal carcinomas of the breast. AJR Am J Roentgenol 189(6):1288-1293

Yeatman TJ, Cantor AB, Smith TJ, Smith SK, Reintgen DS, Miller MS, Ku NN, Baekey PA, Cox CE (1995) Tumor biology of infiltrating lobular carcinoma. Implications for management. Ann Surg 222(4):549-559

Yeh ED, Slanetz PJ, Edmister WB, Talele A, Monticciolo D, Kopans DB (2003) Invasive lobular carcinoma: spectrum of enhancement and morphology on magnetic resonance imaging. Breast J 9(1):13-18

doi:10.1186/2193-1801-2-621

Cite this article as: Menezes et al: Invasive ductolobular carcinoma of the breast: spectrum of mammographic, ultrasound and magnetic resonance imaging findings correlated with proportion of the lobular component. SpringerPlus 2013 2:621.

\section{Submit your manuscript to a SpringerOpen ${ }^{\odot}$ journal and benefit from:}

- Convenient online submission

- Rigorous peer review

- Immediate publication on acceptance

- Open access: articles freely available online

- High visibility within the field

- Retaining the copyright to your article 